\title{
ON SPECTRA OF EXPANSION GRAPHS AND MATRIX POLYNOMIALS, II*
}

\author{
K.-H. FÖRSTER ${ }^{\dagger}$ AND B. NAGY \\ Paper presented at the International Linear Algebra Conference, Haifa, June 2001 \\ Abstract. An expansion graph of a directed weighted graph $G_{0}$ is obtained from $G_{0}$ by replacing \\ some edges by disjoint chains. The adjacency matrix of an expansion graph is a partial linearization of \\ a matrix polynomial with nonnegative coefficients. The spectral radii for different expansion graphs \\ of $G_{0}$ and correspondingly the spectral radii of matrix polynomials with nonnegative coefficients, \\ which sum up to a fixed matrix, are compared. A limiting formula is proved for the sequence of the \\ spectral radii of a sequence of expansion graphs of $G_{0}$ when the lengths of all chains replacing some \\ original edges tend to infinity. It is shown that for all expansion graphs of $G_{0}$ the adjacency matrices \\ have the same level characteristic, but they can have different height characteristics as examples \\ show.
}

Key words. Expansion graph, Matrix polynomial, Graph spectrum, Height characteristic, Level characteristic.

AMS subject classifications. 05C50, 15A22, 15A42, 05C20

1. Introduction. In [7] S. Friedland and H. Schneider defined the concept of expansion graph for an unweighted directed graph, which is obtained from the given graph by replacing certain edges by (exactly) one chain; they studied the effect of graph expansions on the spectrum (of the adjacency matrix) of the graph. In [6] we generalized this concept to weighted graphs by replacing certain edges of the given graph by possibly several disjoint chains of different lengths. One essential observation was that the nonzero spectrum of the adjacency matrix of an expansion graph coincides with the nonzero spectrum of a matrix polynomial with nonnegative coefficients [6, Prop. 1, Th. 6]. We used this fact to study the peripheral spectrum if the given graph is connected. In this article we consider arbitrary weighted digraphs and study especially the influence of graph expansions on the spectral radius of the adjacency matrix.

The paper contains four further sections. In the next section we give the definitions and collect results by different authors used in the later sections. In section 3 we compare the spectral radii of the adjacency matrices of different expansion graphs of a given graph or equivalently we compare the spectral radii of different matrix polynomials with constant sum of their coefficients. In section 4 we consider a sequence of expansion graphs of a given graph. We prove that the sequence of the corresponding spectral radii converges if the lengths of all chains replacing the original edges in a

${ }^{*}$ Received by the editors on 30 November 2001. Final version accepted for publication on 1 July 2002. Handling Editor: Hans Schneider.

$\dagger$ Department of Mathematics, Technical University Berlin, Sekr. MA 6-4, Strasse des 17. Juni 136, D-10623 Berlin, Germany (foerster@math.tu-berlin.de).

${ }^{\ddagger}$ Department of Analysis, Institute of Mathematics, Technical University of Budapest, H-1521 Budapest, Hungary (bnagy@math.bme.hu). This author's work was supported by the Hungarian OTKA Grant No. T030042. 
certain subset $F$ of the set of all original edges tend to infinity, and the graphs of the sequence coincide on the complement of $F$ with a fixed expansion graph. In section 5 we study the connections between the reduced graph of a graph expansion and the reduced graph of the expanded graph. We show that the level characteristics (for the spectral radius) of all expansion graphs of a given graph with spectral radius one are identical, but we give examples of graph expansions of a given graph with different height characteristics (for the spectral radius).

2. Results on expansion graphs and matrix polynomials. Our work relies heavily on the work of [6], [7] and of other authors so in this section we set the notation and we describe known results on expansion graphs needed for the next sections.

Let $S$ be a nonnegative $n \times n$ matrix and let $G(S)$ be the directed weighted graph with adjacency matrix $S$, i.e., with $\langle n\rangle=\{1, \ldots, n\}$,

$$
\begin{aligned}
G(S) & =(V(S), E(S)) \\
& =(\langle n\rangle,\{(i, j) \in\langle n\rangle \times\langle n\rangle: S(i, j) \neq 0\}),
\end{aligned}
$$

and the edge $(i, j) \in E(S)$ has weight $S(i, j)$; therefore the "edge" $(i, j) \in\langle n\rangle \times$ $\langle n\rangle \backslash E(S)$ has weight zero as usual. Here $S(i, j)$ denotes the element in the $i$ th row and the $j$ th column of the matrix $S$.

Let $A_{0}, \ldots, A_{l}$ be nonnegative $n \times n$ matrices with $A_{0}+\ldots+A_{l}=S$. The expansion graph $G\left(A_{0}, \ldots, A_{l}\right)$ of $G(S)$ with respect to $\left(A_{0}, \ldots, A_{l}\right)$ is the directed, weighted graph defined as follows. The set $V\left(A_{0}, \ldots, A_{l}\right)$ of its vertices is given by the following procedure: For each triple $(i, j, k) \in\langle n\rangle \times\langle n>\times\{0,1, \ldots, l\}$ with $A_{k}(i, j) \neq 0$ add $l-k$ "additional" vertices to $\langle n\rangle$, labeled by

$$
(i, j, k, p), \quad p=1,2, \ldots, l-k
$$

further, define $(i, j, k, 0)=i$ and $(i, j, k, l-k+1)=j$. The set $E\left(A_{0}, \ldots, A_{l}\right)$ of its edges is given by

$$
\begin{aligned}
& ((i, j, k, p),(\hat{\imath}, \hat{\jmath}, \hat{k}, \hat{p})) \in E\left(A_{0}, \ldots, A_{l}\right) \text { if and only if } \\
& (\hat{\imath}, \hat{\jmath}, \hat{k}, \hat{p})=(i, j, k, p+1) \text { in case } p \neq 0 \text { and } \hat{p} \neq l-\hat{k}+1 \\
& (i,(\hat{\imath}, \hat{\jmath}, \hat{k}, \hat{p})) \in E\left(A_{0}, \ldots, A_{l}\right) \text { if and only if } \\
& \left.A_{\hat{k}}(i, \hat{\jmath}) \neq 0, i=\hat{\imath}=(\hat{\imath}, \hat{\jmath}, \hat{k}, 0)\right), \text { and } \hat{p}=1 \\
& ((i, j, k, p), \hat{\jmath}) \in E\left(A_{0}, \ldots, A_{l}\right) \text { if and only if } \\
& A_{k}(i, j) \neq 0, \hat{\jmath}=j=(i, j, k, l-k+1), \text { and } p=l-k
\end{aligned}
$$


The weights of the edges of $G\left(A_{0}, \ldots, A_{l}\right)$ will be chosen so that

$$
\prod_{p=0}^{l-k} w((i, j, k, p),(i, j, k, p+1))=A_{k}(i, j)
$$

$$
\text { for }(i, j, k) \in\langle n\rangle \times\langle n\rangle \times\{0, \ldots, l\} \text {. }
$$

It follows that

$$
V\left(A_{0}, \ldots, A_{l}\right)=\langle n\rangle \cup \bigcup_{k=0}^{l-1}\left\{(i, j, k, p): A_{k}(i, j) \neq 0, p=1, \ldots, l-k\right\} .
$$

The adjacency matrix $A$ of $G\left(A_{0}, \ldots, A_{l}\right)$ is a partial linearization of the monic matrix polynomial $L$ of degree $l+1$ given by

$$
L(z)=z^{l+1}-z^{l} A_{l}-\ldots-z A_{1}-A_{0} ;
$$

see $[6$, Th. 6]. With $L$ we associate the rational matrix function

$$
S_{L}(z)=A_{l}+z^{-1} A_{l-1}+\ldots+z^{-l} A_{0} \quad \text { for } z \neq 0 .
$$

Then we have $L(z)=z^{l}\left(z-S_{L}(z)\right) \quad$ for $z \neq 0 \quad$ and $S_{L}(1)=S$.

S. Friedland and H. Schneider considered in [7] the special case that all $A_{k}$ and $S$ are unimodular matrices; with their terminology we have

$$
\begin{aligned}
& l=\max \{w(i, j):(i, j) \in E(S)\}, \text { and for }(i, j) \in E(S): \\
& A_{k}(i, j)=1 \text { if } w(i, j)=l-k, \text { and } A_{k}(i, j)=0 \text { if } w(i, j) \neq l-k, \\
& \text { and } \\
& A_{k}(i, j)=0 \text { for all } k=0,1, \ldots, l \text { if }(i, j) \in\langle n\rangle \times\langle n\rangle E(S) .
\end{aligned}
$$

The auxiliary matrix $A_{w}$ in [7, Def. 2.1] is identical with $S_{L}$ above.

As usual, the spectrum of a square matrix $A$ is the set of its eigenvalues, and is denoted by $\operatorname{spec}(A)$. The spectral radius of $A$ is the largest absolute value of its eigenvalues, and is denoted by $r(A)$. For a monic matrix polynomial $L$ its spectrum $\operatorname{spec}(L)$ is the set $\{z \in \mathbb{C}: L(z)$ is singular $\}$ and its spectral radius $r(L)$ is the largest absolute value of the elements of $\operatorname{spec}(L)$. If $A$ is the adjacency matrix of a graph $G$ we write $\operatorname{sometimes} \operatorname{spec}(A)$ as $\operatorname{spec}(G)$ and $r(A)$ as $r(G)$.

In the following theorem we collect known results on expansion graphs and on spectral properties of monic matrix polynomials with nonnegative coefficients.

THEOREM 2.1. Let $A_{0}, \ldots A_{l}$ be nonnegative $n \times n$ matrices and set $S=A_{0}+$ $\ldots+A_{l}$. With the notations from above we have:

(i) [6, Th. 6] The adjacency matrix $A$ of the expansion graph $G\left(A_{0}, \ldots, A_{l}\right)$ is cogredient to a block matrix

$$
\left[\begin{array}{cc}
N & H \\
G & A_{l}
\end{array}\right] \text { with } N^{l}=0 \text { and } A_{k-1}=G N^{l-k} H \text { for all } k=1, \ldots, l \text {. }
$$


(ii) $\left[6\right.$, Prop. 1] $\operatorname{spec}(A) \backslash\{0\}=\operatorname{spec}(L) \backslash\{0\}$, especially $r(A)=r\left(G\left(A_{0}, \ldots, A_{l}\right)\right)$ $=r(L)$.

(iii) If $z \neq 0$, then $\operatorname{ker}(L(z))=\operatorname{ker}\left(z-S_{L}(z)\right)$, therefore $z \in \operatorname{spec}(L)$ and $z \in$ $\operatorname{spec}\left(S_{L}(z)\right)$ are equivalent.

(iv) [10, Prop. 2.1] The function $] 0, \infty\left[\rightarrow \mathbb{R}_{+}, t \mapsto r\left(S_{L}(t)\right)\right.$ is nonincreasing and continuous. If $r\left(S_{L}(t)\right)>0$ for some $\left.t \in\right] 0, \infty\left[\right.$, then $r\left(S_{L}(t)\right)>0$ for all $t \in] 0, \infty[$, and there exists at most one $t \in] 0, \infty\left[\right.$ with $r\left(S_{L}(t)\right)=t$.

(v) [3, Theorem] and [10, Prop. 2.1] If $t>0$, then $r(L)=t$ and $r\left(S_{L}(t)\right)=t$ are equivalent.

(vi) [10, Prop. 2.1] The assertions $r(S)=0, r(L)=0$ and $r\left(S_{L}(t)\right)=0$ for all $t \in] 0, \infty[$ are equivalent.

Special cases of these results can be found in [7].

3. Comparison of the spectral radii of matrix polynomials and of the adjacency matrices of expansion graphs. Throughout this section we assume that the assumptions of section 1 hold and we use the notations of section 1 . First we investigate the relation of the spectral radii $r(A)=r(L)$ and $r(S)$. We extend slightly the assertions of [3, Prop. 1.1].

Proposition 3.1. Let $A_{0}, \ldots, A_{l}$ be nonnegative square matrices and let $S=$ $A_{0}+\ldots+A_{l}$. Then

$$
1 \leq r(S) \Rightarrow r(S)^{1 /(l+1)} \leq r(L) \leq r(S)
$$

and

$$
r(S) \leq 1 \Rightarrow r(S) \leq r(L) \leq r(S)^{1 /(l+1)}
$$

If $r(S)>0$, then the reverse implications hold in both cases.

Proof. Theorem 2.1 (v) and (vi) show that in the cases $r(S)=0$ and $r(S)=1$ there is nothing to prove.

Let $r(S)>1$. Then by Theorem $2.1(\mathrm{v})$

$$
r\left(S_{L}(t)\right) \geq r(S)>1 \geq t, \quad 0<t \leq 1 .
$$

By Theorem 2.1 (iv) and (v) we obtain $r(L)>1$. It is easy to show (see the proof of [10, Prop. 2.1]) that for $0<t_{1} \leq t_{2}$

$$
0 \leq\left(\frac{t_{1}}{t_{2}}\right)^{l} S_{L}\left(t_{1}\right) \leq S_{L}\left(t_{2}\right) \leq S_{L}\left(t_{1}\right) \leq\left(\frac{t_{2}}{t_{1}}\right)^{l} S_{L}\left(t_{2}\right)
$$

holds. These inequalities imply

$$
\left(\frac{t_{1}}{t_{2}}\right)^{l} r\left(S_{L}\left(t_{1}\right)\right) \leq r\left(S_{L}\left(t_{2}\right)\right) \leq r\left(S_{L}\left(t_{1}\right)\right) \leq\left(\frac{t_{2}}{t_{1}}\right)^{l} r\left(S_{L}\left(t_{2}\right)\right) .
$$

Set $t_{1}=1$ and $t_{2}=r(L)$. Then by Theorem $2.1(\mathrm{v})$ we obtain $r(S) \leq r(L)^{l+1}$ and $r(L) \leq r(S)$. 
The second implication is proved similarly. The last assertion is trivial.

REMARK 3.2. Using Theorem 2.1 (ii) we see that Proposition 3.1 generalizes the first part of [7, Th. 3.5] for expansion graphs. The second part of [7, Th. 3.6] will be generalized in the last proposition of this section.

Proposition 3.3. Let $l$ be a nonnegative integer and $S \geq 0$. Then for all $t$ in the closed interval

$$
\left[\min \left\{r(S), r(S)^{1 /(l+1)}\right\}, \max \left\{r(S), r(S)^{1 /(l+1)}\right\}\right]
$$

there exist nonnegative square matrices $A_{0}, \ldots, A_{l}$ such that $S=A_{0}+\ldots+A_{l}$ and $r(L)=t$.

Proof. For $0 \leq a \leq 1$ set $L_{a}(z)=z^{l+1}-z^{l} a S-(1-a) S$. Then $r\left(L_{0}\right)=r(S)^{1 / l+1}$ and $r\left(L_{1}\right)=r(S)$. The function $[0,1] \rightarrow \mathbb{R}_{+}$with $a \longmapsto r\left(L_{a}\right)$ is continuous. Apply the intermediate value theorem to obtain the assertion.

Proposition 3.4. Let $S \geq 0$. Then for all $t$ in the half-open interval

$$
] \min \{1, r(S)\}, \max \{1, r(S)\}[\cup\{r(S)\}
$$

there exists a nonnegative integer $l$ and nonnegative square matrices $A_{0}, \ldots A_{l}$ such that $S=A_{0}+\ldots+A_{l}$ and $r(L)=t$.

Proof. Let $t=r(S)$. Then choose $l=0$ and $A_{0}=S$. If $r(S)>1$ and $\left.t \in\right] 1, r(S)[$, then choose $l$ such that $r(S)^{1 /(l+1)} \leq t$ and apply Proposition 3.2. The remaining case $r(S)<1$ can be proved similarly.

TheOREM 3.5. Let $S \geq 0$ and let $l$ and $m$ be nonnegative integers with $l \leq m$. Further let $A_{0}, A_{1}, \ldots, A_{l}$ and $B_{0}, B_{1}, \ldots, B_{m}$ be nonnegative matrices with $A_{0}+A_{1}+$ $\ldots+A_{l}=S=B_{0}+B_{1}+\ldots+B_{m}$. Set

$$
\begin{aligned}
& L(z)=z^{l+1}-z^{l} A_{l}-\ldots-z A_{1}-A_{0}, \quad \text { and } \\
& M(z)=z^{m+1}-z^{m} B_{m}-\ldots-z B_{1}-B_{0},
\end{aligned}
$$

and assume that

$$
\sum_{h=0}^{k} A_{h} \leq \sum_{h=0}^{k+m-l} B_{h} \quad \text { for all } \quad k=0, \ldots, l-1
$$

hold. Then

$$
\begin{aligned}
& 1 \leq r(S) \Rightarrow 1 \leq r(M) \leq r(L), \quad \text { and } \\
& r(S) \leq 1 \Rightarrow r(L) \leq r(M) \leq 1
\end{aligned}
$$

Proof. Let $\tilde{L}(z)=z^{m-l} L(z)$. Then $S_{\tilde{L}}=S_{L}$ and $r(\tilde{L})=r(L)$. Apply [2, Lemma $3]$ to $\tilde{L}$ and $M$ to obtain the result. For the convenience of the reader we indicate a proof [5, Cor. 3.5]. 
Let $z \neq 0$. Then

$$
\begin{aligned}
S_{M}(z)=S+\left(\frac{1}{z}\right. & -1)\left(B_{m-1}+\ldots+B_{0}\right)+\ldots \\
& +\left(\frac{1}{z^{l}}-\frac{1}{z^{l-1}}\right)\left(B_{m-l}+\ldots+B_{0}\right)+\ldots+\left(\frac{1}{z^{m}}-\frac{1}{z^{m-1}}\right) B_{0} .
\end{aligned}
$$

A similar expression for $S_{L}(z)$ and the inequalities (3.3) show that $S_{M}(t) \leq S_{L}(t)$ for $t \geq 1$. Assume $r(S) \geq 1$. Then $r(L) \geq 1$ and $r(M) \geq 1$ by Proposition 3.1. From Theorem 2.1 (iv) and (v) we obtain $r\left(S_{M}(r(L))\right) \leq \bar{r}\left(S_{L}(r(L))\right)=r(L)$ and $r(M) \leq r(L)$.

The proof of the second implication is similar.

REMARK 3.6. In the case of [7] it follows easily that the set of the inequalities (3.3) is equivalent to $w \leq w^{\prime}$, where $w$ induces the expansion graph $G\left(A_{0}, \ldots, A_{l}\right)$ and $w^{\prime}$ induces the expansion graph $G\left(B_{0}, \ldots B_{m}\right)$ of $G(S)$, respectively. Therefore the theorem above generalizes [7, Th. 3.6].

From the theorem above we obtain the following extension of Proposition 3.1.

Corollary 3.7. Let $L(z)=z^{l+1}-z^{l-p} A_{l-p}-\ldots-z^{q} A_{q}$, where $p$ and $q$ are integers with $0 \leq q \leq l-p \leq l$, i.e., $A_{0}=\ldots=A_{q-1}=A_{l-p+1}=\ldots=A_{l}=0$. Then

$$
\begin{aligned}
& 1 \leq r(S) \Rightarrow 1 \leq r(S)^{\frac{1}{l-q+1}} \leq r(L) \leq r(S)^{\frac{1}{p+1}} \\
& r(S) \leq 1 \Rightarrow r(S)^{\frac{1}{p+1}} \leq r(L) \leq r(S)^{\frac{1}{l-q+1}} \leq 1 .
\end{aligned}
$$

Proof. Apply the theorem first to $\tilde{M}=L$ and $\tilde{L}$, where $\tilde{L}(z)=z^{l+1}-z^{l-q} S$ and secondly to $L$ and $M$, where $M(z)=z^{l+1}-z^{q} S$.

REMARK 3.8. The condition

$$
A_{0}=\ldots=A_{q-1}=0=A_{l-p+1}=\ldots=A_{l}=0
$$

is equivalent to: For all $(i, j) \in E(S)$ the lengths of all paths from $i$ to $j$ in the expansion graph $G\left(A_{0}, \ldots, A_{l}\right)$ are less than $l-q$ and greater than $p$. Therefore the corollary generalizes [7, Cor. 3.7].

Corollary 3.9. If $S$ is irreducible, $r(S) \neq 0$ and at least one of the inequalities in (3) is proper, then $r(L) \neq r(M)$.

Proof. The assumptions imply $r(S)>0$; for $r(S)=0$ and $S$ is irreducible imply that $S$ is the $1 \times 1$ zero matrix. Then all $A_{i}$ and all $B_{j}$ are the $1 \times 1$ zero matrix, and we have equality in (3.3) for all $k=0, \ldots, l-1$. For all $t>0$ the matrices $S_{L}(t)$ and $S_{M}(t)$ are irreducible (cf. $\left.(3.1)\right), S_{L}(t) \lesseqgtr S_{M}(t)$ for all $\left.t \in\right] 0,1\left[\right.$ and $S_{M}(t) \lesseqgtr S_{L}(t)$ for all $t \in] 1, \infty[$ (cf. proof of Theorem 4). By [9, Th.2.2] this implies $r(L) \neq r(M)$.

Proposition 3.10. Let $S$ be irreducible. Then $r(L)=r(S)$ if and only if $S=A_{l}$ or $r(S)=1$.

Proof. It is clear that each of the conditions $S=A_{l}$ or $r(S)=1$ implies $r(L)=$ $r(S)$; see Proposition 3.1. 
If $r(S)=0$ then $S$ and $A_{l}$ are the $1 \times 1$ zero matrix. Let $0<r=r(S)$. We will prove that $S \neq A_{l}$ and $r(L)=r(S) \neq 1$ lead to a contradiction. Since $S$ is irreducible there exists a strictly positive eigenvector $x$ of $S$ to $r$. The adjacency matrix $A=\left[\begin{array}{cc}N & H \\ G & A_{l}\end{array}\right]$ of $G\left(A_{0}, \ldots, A_{l}\right)$ is irreducible and $r(A)=r(L)$; see [6, Prop. 3 and 8]. Define

$$
u=\left[\begin{array}{c}
(r-N)^{-1} H x \\
x
\end{array}\right]
$$

Then

$$
0 \lesseqgtr u \text { and } A u=\left[\begin{array}{c}
r(r-N)^{-1} H x \\
S_{L}(r) x
\end{array}\right] .
$$

Let $0<r=r(S)<1$ and $S \neq A_{l}$. Then $S_{L}(r) x \supsetneqq S x=r x$ and therefore $A u \supsetneqq$ $r u$. But this is false; $A$ is irreducible and $r=r(L)=r(A)$, therefore the range of $r-A$ does not contain nonzero nonnegative and nonzero nonpositive vectors; see the considerations on p.12 in [9].

Let $1<r=r(S)$ and $S \neq A_{l}$. Then $S_{L}(r) x \lessgtr S x=r x$ and therefore $A u \lessgtr r u$. Again this is false for the same reason.

REMARK 3.11. For expansion graphs this proposition means: Let $G(S)$ be connected. Then for every expansion graph of $G(S)$ with adjacency matrix $A, r(A)=r(S)$ is equivalent to the statement that the expansion graph coincides with $G(S)$ or $r(S)=1$.

4. Limiting cases. From Proposition 3.3 it follows that the spectral radii (of the adjacency matrices) of all expansion graphs of $G(S)$ lie in a bounded interval. In this section we will give conditions for a sequence of expansion graphs of $G(S)$ which imply that the sequence of their spectral radii converges.

DeFinition 4.1. Let $S$ be a nonnegative $n \times n$ matrix and $G(S)=(V(S), E(S))$ be the graph induced by $S$. Let $\left(G_{m}\right)_{m=1}^{\infty}=\left(G\left(A_{m, 0}, \ldots, A_{m, l_{m}}\right)\right)_{m=1}^{\infty}$ be an infinite sequence of expansion graphs of $G(S)$. Let $F \subset E(S)$. We say that $\left(G_{m}\right)_{m=1}^{\infty}$ tends to infinity on $F$ if for all $(i, j) \in F$ the lengths of all paths from $i$ to $j$ in $G_{m}$ tend to infinity if $m$ tends to infinity, i.e.,

$$
\lim _{m \rightarrow \infty} \min \left\{h: 0 \leq h \leq l_{m}, A_{m, l_{m}-h}(i, j) \neq 0\right\}=\infty \quad \text { for all } \quad(i, j) \in F .
$$

This concept generalizes immediately [7, Def. 4.2.(ii)].

Proposition 4.2. Let $S$ be a nonnegative square matrix with $r(S)>0$. Let $\left(G_{m}\right)_{m=1}^{\infty}$ be an infinite sequence of expansion graphs of $G(S)$ tending to infinity on $E(S)$. Then $\lim _{m \rightarrow \infty} r\left(G_{m}\right)=1$.

Proof. Let $G_{m}=G\left(A_{m, 0}, \ldots A_{m, l_{m}}\right)$ for all $m=1,2, \ldots$ Define

$$
p_{m}=\min \left\{\min \left\{h: 0 \leq h \leq l_{m}, A_{m, h}(i, j) \neq 0\right\}:(i, j) \in E(S)\right\} .
$$

Then $\lim _{m \rightarrow \infty} p_{m}=\infty$. From Corollary 3.5 we obtain $\left|1-r\left(L_{m}\right)\right| \leq\left|1-r(S)^{\frac{1}{p_{m}+1}}\right|$, where $L_{m}(z)=z^{l_{m}+1}-z^{l_{m}} A_{m, l_{m}}-\ldots-A_{m, 0}$. Since $r\left(L_{m}\right)=r\left(G_{m}\right)$ by Theorem 2.1(ii), $\lim _{m \rightarrow \infty} r\left(G_{m}\right)=1$ follows. 
Definition 4.3. Let $S$ be a nonnegative square matrix. Let $G\left(A_{0}, \ldots, A_{l}\right)$ and $G\left(B_{0}, \ldots, B_{m}\right)$ be expansion graphs of $G(S)$. Let $F \subset E(S)$. We say that $G\left(A_{0}, \ldots, A_{l}\right)$ and $G\left(B_{0}, \ldots, B_{m}\right)$ coincide on $F$ if

$$
A_{l-h}(i, j)=B_{m-h}(i, j) \quad \text { for all } \quad h=0, \ldots, \min \{l, m\} \quad \text { and all } \quad(i, j) \in F .
$$

This definition is a direct generalization of [7, Def. 4.2. (i)]. $G(S)$ and its expansion graph $G\left(A_{0}, \ldots, A_{l}\right)$ coincide on $E(S)$ if and only of $A_{l}=S$ (and then $A_{l-1}=\ldots=$ $\left.A_{0}=0\right)$. The following theorem generalizes [7, Th. 4.3, Cor. 4.4].

THEOREM 4.4. Let $S$ be a nonnegative $n \times n$ matrix with $r(S) \geq 1$. Let $G$ be an expansion graph of $G(S)$ and let $\left(G_{m}\right)_{m=1}^{\infty}$ be an infinite sequence of expansion graphs of $G(S)$. Let $F \subset E(S)$. Assume that $\left(G_{m}\right)_{m=1}^{\infty}$ tends to infinity on $F$, and $G$ and $G_{m}$ coincide on $F^{\prime}=E(S) \backslash F$ for all $m=1,2, \ldots$ For $h=0 \ldots$, let the nonnegative $n \times n$ matrices $A_{h}^{\prime}$ be given by

$$
A_{h}^{\prime}(i, j)= \begin{cases}A_{h}(i, j) & \text { if }(i, j) \in F^{\prime}=E(S) \backslash F \\ 0 & \text { otherwise. }\end{cases}
$$

Let $G^{\prime}$ be the expansion graph $G\left(A_{0}^{\prime} \ldots, A_{l}^{\prime}\right)$ of $G\left(S^{\prime}\right)$ where $S^{\prime}=A_{0}^{\prime}+\ldots+A_{l}^{\prime}$. Then

(i) $r\left(G^{\prime}\right) \leq 1$ implies $\lim _{m \rightarrow \infty} r\left(G_{m}\right)=1$;

(ii) $r\left(G^{\prime}\right) \geq 1$ implies $\lim _{m \rightarrow \infty} r\left(G_{m}\right)=r\left(G^{\prime}\right)$.

Proof. Let $G=G\left(A_{0}, \ldots, A_{l}\right)$ and $G_{m}=G\left(A_{m, 0}, \ldots, A_{m, l_{m}}\right)$ for all $m=1,2, \ldots$ and let $L$ and $L_{m}$ be the corresponding monic matrix polynomials of degree $l+1$ and $l_{m}+1$, respectively.

From Theorem 2.1 (ii) and Proposition 3.1 we obtain $1 \leq r\left(G_{m}\right)=r\left(L_{m}\right) \leq r(S)$ for all $m=1,2 \ldots$

Next we show that $r\left(G^{\prime}\right) \leq r\left(G_{m}\right)$ for all $m=1,2, \ldots$ Set $L^{\prime}(z)=z^{l+1}-z^{l} A_{l}^{\prime}-$ $\ldots-A_{0}^{\prime}$. For all $t>0$, all $(i, j) \in\langle n\rangle \times\langle n\rangle$ and all $m=1,2, \ldots$ we have

$$
\begin{aligned}
& S_{L^{\prime}}(t)(i, j)=S_{L}(t)(i, j)=S_{L_{m}}(t)(i, j) \text { if }(i, j) \in F^{\prime}, \text { and } \\
& S_{L^{\prime}}(t)(i, j)=0 \leq S_{L_{m}}(t)(i, j) \text { if }(i, j) \notin F^{\prime} .
\end{aligned}
$$

This implies $S_{L^{\prime}}(t) \leq S_{L_{m}}(t)$ for all $t>0$ and $m=1,2, \ldots$. By Theorem 2.1 (ii), (iv) and $(\mathrm{v})$, we obtain $r\left(G^{\prime}\right)=r\left(L^{\prime}\right) \leq r\left(L_{m}\right)=r\left(G_{m}\right)$ for all $m=1,2, \ldots$

Now it is clear that the implications (i) and (ii) are true if $\lim _{m \rightarrow \infty} \sup r\left(G_{m}\right)=1$. We will show below that

$$
\lim _{m \rightarrow \infty} \sup r\left(G_{m}\right)>1 \text { implies } \lim _{m \rightarrow \infty} \sup r\left(G_{m}\right) \leq r\left(G^{\prime}\right) ;
$$

this implication completes the proof of the theorem.

For all $t>1$, all $(i, j) \in F$ and all $m=1,2, \ldots$ we obtain

$0 \leq S_{L_{m}}(t)(i, j)=t^{-p_{m}} A_{m, l_{m}-p_{m}}(i, j)+\ldots+t^{-l_{m}} A_{m, 0}(i, j) \leq t^{-p_{m}} S(i, j)$, where $p_{m}=\min \left\{\min \left\{h: 0 \leq h \leq l_{m}, A_{l_{n}, l_{m}-h}(i, j) \neq 0\right\}:(i, j) \in F\right\}$.

This implies $\lim _{m \rightarrow \infty} S_{L_{m}}(t)=S_{L^{\prime}}(t)$ for $t>1$. Therefore $\lim _{m \rightarrow \infty} r\left(S_{L_{m}}(t)\right)=$ $r\left(S_{L^{\prime}}(t)\right)$ for $t>1$. 
Let $r=\lim _{m \rightarrow \infty} \sup r\left(G_{m}\right)>1$ and $\left.q \in\right] 1, r[$. There exists a subsequence

$$
\left(r\left(G_{m(k)}\right)\right)_{k=1}^{\infty} \text { of }\left(r\left(G_{m}\right)\right)_{m=1}^{\infty}
$$

such that $q \leq r\left(G_{m(k)}\right)=r\left(L_{m(k)}\right)$ for all $k=1,2, \ldots$ By Theorem 2.1 (iv) and (v), we get $q \leq r\left(S_{L_{m(k)}}(q)\right)$ for all $k=1,2 \ldots$ Therefore $q \leq r\left(S_{L^{\prime}}(q)\right)$ and then $q \leq r\left(L^{\prime}\right)=r\left(G^{\prime}\right)$ by Theorem 2.1 (ii), (iv) and (v). If $q$ tends to $r$ we obtain $\lim _{m \rightarrow \infty} \sup r\left(G_{m}\right)=r \leq r\left(G^{\prime}\right)$.

5. Level and height characteristics for graph expansions. Let $G=G(S)$ $=(V, E)$ be a weighted digraph with adjacency matrix $S$. The reduced graph $R(G)=$ $R(S)$ of $G(S)$ is defined to be the digraph with the components of $G$ (i.e., the maximal connected subgraphs of $G$ ) as vertices, and there is an edge from one component $\gamma$ to another component $\delta$ in $R(G)$ if there exist vertices $i \in \gamma$ and $j \in \delta$ such that $(i, j) \in E . R(G)$ is unweighted. Let $i$ and $j$ be two vertices in a graph $G=(V, E)$. We say that $i$ has access to $j$ in $G$, if there exists a path in $G$ starting in $i$ and terminating in $j$; i.e., there exists an integer $k \geq 1$ and $i_{0}, i_{1}, \ldots, i_{k}$ in $V$ such that $i_{0}=i, i_{k}=j$ and $\left(i_{m-1}, i_{m}\right) \in E$ for $m=1, \ldots, k$.

Proposition 5.1. Let $G_{\exp }=G\left(A_{0}, \ldots, A_{l}\right)=\left(V_{\exp }, E_{\exp }\right)$ be an expansion graph of $G=G(S)=(V, E)$. Then the following holds:

(i) For a component $\Gamma$ of $G_{\exp }$ the following two assertions are equivalent:

(a) $\Gamma \cap V=\emptyset$,

(b) $\Gamma=\{(i, j, k, p)\}$ for some $i, j, k$ and $p$ with $(i, j) \in E, 0 \leq k \leq l, A_{k}(i, j)$ $\neq 0,0<p \leq l-k$ and $j$ has no access to $i$ in $G$.

(ii) Let $\Gamma$ be a component of $G_{\exp }$ with $\Gamma \cap V \neq \emptyset$. Then $\Gamma \cap V$ is a component of $G$, and it coincides with any component of $G$ which has nonempty intersection with $\Gamma$.

(iii) Let $\gamma$ be a component of $G$. Then $\gamma \cup\left\{(i, j, k, p) \in V_{\exp }: i, j \in \gamma, 0 \leq k \leq\right.$ $\left.l, A_{k}(i, j) \neq 0,0<p \leq l-k\right\}$ is a component of $G_{\exp }$, and it coincides with any component of $G_{\exp }$ which has a nonempty intersection with $\gamma$.

(iv) Let $\gamma$ and $\delta$ be components of $G$ and let $\Gamma$ and $\Delta$ be the uniquely determined components of $G_{\exp }$ with $\gamma \subset \Gamma$ and $\delta \subset \Delta$, respectively. Then $\gamma$ has access to $\delta$ in the reduced graph $R(G)$ if and only if $\Gamma$ has access to $\Delta$ in the reduced graph $R\left(G_{\exp }\right)$.

Proof. We recall that $V \subset V_{\text {exp }}$, and that $(i, j, k, p) \in V_{\exp }$ if and only if $(i, j) \in$ $E, 0 \leq k \leq l, A_{k}(i, j) \neq 0$ and $0 \leq p \leq l-k+1$. The statements in (iii), (a) - (c) before Theorem 6 in Section 3 of [6] imply the following three statements: Let $i, j, \hat{\imath}$ and $\hat{\jmath}$ be contained in $\mathrm{V}$, then

(a) $i$ and $j$ are contained in one component of $G$ if and only if they are contained in one component of $G_{\text {exp. }}$.

(b) Let $(i, j, k, p) \in V_{\exp }$ with $0<p \leq l-k$ and let $\hat{\imath} \in V$. Then these vertices are contained in a component $\Gamma$ of $G_{\exp }$ if and only if $i, j$ and $\hat{\imath}$ are contained in $\Gamma$. This holds if and only if $i, j$ and $\hat{\imath}$ are contained in one component of $G$ (which is $\Gamma \cap V)$.

(c) Let $(i, j, k, p)$ and $(\hat{\imath}, \hat{\jmath}, \hat{k}, \hat{p})$ be different vertices of $G_{\exp }$ with $0<p \leq l-k$ and $0<\hat{p} \leq l-\hat{k}$, respectively. Then these vertices are contained in a component 
$\Gamma$ of $G_{\exp }$ if and only if $i, j, \hat{\imath}, \hat{\jmath}$ are contained in $\Gamma$. This holds if and only if $i, j, \hat{\imath}, \hat{\jmath}$ are contained in one component of $G$ (which is $V \cap \Gamma$ ).

From these statements the first 3 statements of the proposition follow immediately. Two components of a graph have access in the corresponding reduced graph if and only if there are vertices in the components which have access in the graph itself; now (iv) is clear.

Let $A$ be a nonnegative $n \times n$ matrix and let $\gamma \subset\langle n\rangle$. By $A[\gamma]$ we denote the principal submatrix of $A$ lying in the rows and columns indexed by $\gamma$. It is well known that $\gamma \subset G(A)$ is connected in $G(A)$ if and only if $A[\gamma]$ is irreducible (see $[8,4.2]$ ).

Let $G=(V, E)$ be a graph and $\gamma \subset V$. By $s G(\gamma, G)$ we denote the subgraph of $G$ induced by $\gamma$, i.e.

$$
s G(\gamma, G)=(\gamma, E(\gamma, G)=\{(i, j) \in E: i, j \in \gamma\}) .
$$

It follows that $\operatorname{Adj}(s G(\gamma, G))=\operatorname{Adj}(G)[\gamma]$.

Proposition 5.2. Let $G_{\exp }=G\left(A_{0}, \ldots, A_{l}\right)$ be an expansion graph of $G=$ $G(S)=(\langle n\rangle, E)$ for some $n \in \mathbb{N}$. Let $\gamma$ be a component of $G$ and $\Gamma$ the corresponding component of $G_{\exp }$ with $\gamma \subset \Gamma$ (see Proposition 5.1). Then $s G(\gamma, G)=$ $G(S[\gamma]), \quad s G\left(\Gamma, G_{\exp }\right)$ is an expansion graph of $s G(\gamma, G)$ and $s G\left(\Gamma, G_{\exp }\right)=$ $G\left(A_{0}[\gamma], \ldots, A_{l}[\gamma]\right)$.

The proof of this proposition is straightforward.

Let $A$ be a nonnegative $n \times n$ matrix. A vertex $\gamma$ of the reduced graph $R(A)$ is called a basic vertex for $A$ if $r(A[\gamma])=r(A)$; see [11]. Sometimes such a vertex is also called an $r(A)$-vertex for $A$; see [12].

For the next proposition note that for a matrix polynomial $L$ with nonnegative coefficients the reduced graph $R\left(S_{L}(t)\right)$ is independent of $\left.t \in\right] 0, \infty[$; see (3.1).

Proposition 5.3. Let $G_{\exp }=G\left(A_{0}, \ldots, A_{l}\right)$ be an expansion graph of $G(S)$ and let $L(z)=z^{l+1}-z^{l} A_{l}-\ldots-A_{0}$. Assume $r=r\left(G_{\exp }\right)=r\left(S_{L}(r)\right)=r(L)>0$. Let $\gamma$ be a vertex in $R\left(S_{L}(r)\right)=R(S)$ and let $\Gamma$ be the corresponding vertex in $R\left(G_{\exp }\right)$ with $\gamma \subset \Gamma$ (see Proposition 5.1). Then $\gamma$ is a basic vertex for $S_{L}(r)$ if and only if $\Gamma$ is a basic vertex for $\operatorname{Adj}\left(G_{\exp }\right)$.

Proof. Let $\gamma$ be a basic vertex for $S_{L}(r)$. Then

$$
\begin{aligned}
r\left(\operatorname{Adj}\left(G_{\text {exp }}\right)\right) & =r(L)=r=r\left(S_{L}(r)\right) & & \text { by Theorem } 2.1 \\
& =r\left(S_{L}(r)[\gamma]\right) & & \text { by assumption } \\
& =r\left(S_{L[\gamma]}(r)\right)=r(L[\gamma]) & & \text { by definition of } L[\gamma] \\
& =r\left(G\left(A_{0}[\gamma], \ldots, A_{l}[\gamma]\right)\right. & & \text { by Theorem } 2.1 \\
& =r\left(s G\left(\Gamma, G_{\text {exp }}\right)\right) & & \text { by Proposition } 5.2 \\
& =r\left(\operatorname{Adj}\left(G_{\exp }\right)[\Gamma]\right) . & &
\end{aligned}
$$

The reverse implication follows by a similar argument.

Let $A$ be a nonnegative square matrix. The level of a basic vertex $\gamma$ in the reduced graph $R(A)$ is the maximum of the numbers of basic vertices on a path in $R(A)$ that terminates in $\gamma$. Let $m$ be the maximal level of the basic vertices in $R(A)$. The level characteristic $\lambda(A)$ of $A$ is defined to be the sequence $\left(\lambda_{1}, \ldots, \lambda_{m}\right)$, where $\lambda_{k}$ is the number of basic vertices of $R(A)$ of level $k$. 
We summarize our considerations from above in the following theorem.

THEOREM 5.4. Let $S$ be a nonnegative square matrix with positive spectral radius $r(S)$ and let $r \in] \min \{1, r(S)\}, \max \{1, r(S)\}[\cup\{r(S)\}$ (see Proposition 3.3). Then for every finite sequence $\left(A_{0}, \ldots, A_{l}\right)$ of nonnegative matrices with $A_{0}+\ldots+A_{l}=S$ and $r=r(L)=r\left(S_{L}(r)\right)=r\left(G_{\exp }\right)$ the matrices $S_{L}(r)$ and $\operatorname{Adj}\left(G_{\exp }\right)$ have the same level characteristics; here $L$ denotes the monic polynomical $L(z)=z^{l+1}-z^{l} A_{l}-\ldots-A_{0}$ and $G_{\exp }$ denotes the expansion graph $G\left(A_{0}, \ldots, A_{l}\right)$ of $G(S)$.

In particular: The level characteristics of the adjacency matrices of all expansion graphs of a given weighted digraph with spectral radius 1 are identical.

For a nonnegative square matrix $A$ let $p$ be the maximal positive integer such that $\operatorname{dim} \operatorname{ker}\left((r(A)-A)^{p}\right)>\operatorname{dim} \operatorname{ker}\left((r(A)-A)^{p-1}\right)$. This number is called the index of $A$ or the ascent or the descent of $A$ to its spectral radius $r(A)$. It is equal to maximum of the lengths of the nontrivial Jordan chains of $A$ to $r(A)$, to the maximum of the orders of the Jordan blocks of $A$ to $r(A)$ and to the order of the pole $r(A)$ of the resolvent of $A$.

The height characteristic $\eta(A)$ of $A$ is defined to be the sequence $\left(\eta_{1}, \ldots, \eta_{p}\right)$, where $\eta_{k}=\operatorname{dim} \operatorname{ker}\left((r(A)-A)^{k}\right)-\operatorname{dim} \operatorname{ker}\left((r(A)-A)^{k-1}\right) . \eta(A)$ is nonincreasing.

From the Nonnegative Basis Theorem in [11, Th. 3.1] and other results see [8, p. 181] it follows that the level and the height characteristics of a nonnegative matrix have the same lengths and $\eta(A)$ majorizes $\lambda(A)$, i.e., $\hat{\lambda}_{1}+\ldots+\hat{\lambda}_{k} \leq \eta_{1}+\ldots+\eta_{k}$ for all $k \leq p$ and equality for $k=p$, where $\hat{\lambda}(A)=\left(\hat{\lambda}_{1}, \ldots, \hat{\lambda}_{p}\right)$ denotes the sequence $\lambda(A)$ reordered in a nonincreasing order; see [8, Th. 4.2].

Let the assumptions of Theorem 5.4 hold so that $\lambda\left(S_{L}(r)\right)=\lambda\left(\operatorname{Adj}\left(G_{\text {exp }}\right)\right)$. From [6, Cor. 2.2] and Theorem 2.1(iii) we obtain

$$
\begin{aligned}
\eta_{1}\left(S_{L}(r)\right) & =\operatorname{dim} \operatorname{ker}\left(r-S_{L}(r)\right) \\
& =\operatorname{dim} \operatorname{ker}(L(r)) \\
& =\operatorname{dim} \operatorname{ker}\left(r-\operatorname{Adj}\left(G_{\exp }\right)\right) \\
& =\eta_{1}\left(\operatorname{Adj}\left(G_{\text {exp }}\right)\right) .
\end{aligned}
$$

¿From [3, Theorem 4] we obtain

$$
\begin{aligned}
\eta_{1}\left(S_{L}(r)\right)+\ldots+\eta_{p}\left(S_{L}(r)\right) & =\operatorname{dim} \operatorname{ker}\left(\left(r-S_{L}(r)^{p}\right)\right. \\
& =\operatorname{dim} K(L, r) \\
& =\operatorname{dim} \operatorname{ker}\left(r-\operatorname{Adj}\left(G_{\exp }\right)^{p}\right) \\
& =\eta_{1}\left(\operatorname{Adj}\left(G_{\exp }\right)\right)+\ldots+\eta_{p}\left(\operatorname{Adj}\left(G_{\exp }\right)\right) .
\end{aligned}
$$

Here $K(L, r)$ denotes the space

$$
\left\{x \in \mathbb{C}^{n}: \quad \text { there exists a Jordan chain } x_{0}, \ldots, x_{m}=x \text { of } L \text { to } r\right\} \text {; }
$$

see $[3,(0.3)],\left[6\right.$, Prop. 7]. But we can have $\eta\left(S_{L}(r)\right) \neq \eta\left(\operatorname{Adj}\left(G_{\text {exp }}\right)\right)$ as the following example shows. 
EXAMPle 5.5. Let

$$
A_{1}=\left[\begin{array}{cccccccccccccccc}
0 & 0 & 0 & 0 & 0 & 0 & 0 & 0 & 0 & 0 & 0 & 0 & 0 & 0 & 0 & 0 \\
0 & 1 & 0 & 0 & 0 & 0 & 0 & 0 & 0 & 0 & 0 & 0 & 0 & 0 & 0 & 0 \\
0 & 0 & 1 / 2 & 0 & 0 & 0 & 0 & 0 & 0 & 0 & 0 & 0 & 0 & 0 & 0 & 0 \\
1 & 0 & 0 & 0 & 0 & 0 & 0 & 0 & 0 & 0 & 0 & 0 & 0 & 0 & 0 & 0 \\
0 & 1 & 0 & 0 & 0 & 0 & 0 & 0 & 0 & 0 & 0 & 0 & 0 & 0 & 0 & 0 \\
0 & 0 & 1 & 0 & 0 & 0 & 0 & 0 & 0 & 0 & 0 & 0 & 0 & 0 & 0 & 0 \\
0 & 0 & 0 & 0 & 0 & 0 & 0 & 1 & 0 & 0 & 0 & 0 & 0 & 0 & 0 & 0 \\
0 & 0 & 0 & 0 & 1 & 0 & 0 & 0 & 1 / 2 & 0 & 0 & 0 & 0 & 0 & 0 & 0 \\
0 & 0 & 0 & 1 & 0 & 0 & 0 & 0 & 0 & 0 & 0 & 0 & 0 & 0 & 0 & 0 \\
0 & 0 & 0 & 1 & 1 & 0 & 0 & 0 & 0 & 0 & 0 & 0 & 0 & 0 & 0 & 0 \\
0 & 0 & 0 & 0 & 0 & 0 & 1 & 1 & 5 & 2 & 1 / 2 & 0 & 0 & 0 & 0 & 0 \\
0 & 0 & 0 & 0 & 0 & 0 & 3 & 4 & 1 & 2 & 0 & 0 & 0 & 0 & 0 & 0 \\
0 & 0 & 0 & 0 & 0 & 0 & 2 & 5 & 4 & 1 & 0 & 0 & 1 & 0 & 0 & 0 \\
0 & 0 & 0 & 0 & 0 & 0 & 0 & 0 & 0 & 0 & 1 & 0 & 0 & 1 & 0 & 0 \\
0 & 0 & 0 & 0 & 0 & 0 & 0 & 0 & 0 & 0 & 0 & 1 & 0 & 0 & 0 & 0 \\
0 & 0 & 0 & 0 & 0 & 0 & 0 & 0 & 0 & 0 & 0 & 0 & 1 & 0 & 0 & 0
\end{array}\right]
$$

and

$$
A_{0}=\left[\begin{array}{cccccccccccccccc}
1 & 0 & 0 & 0 & 0 & 0 & 0 & 0 & 0 & 0 & 0 & 0 & 0 & 0 & 0 & 0 \\
0 & 0 & 0 & 0 & 0 & 0 & 0 & 0 & 0 & 0 & 0 & 0 & 0 & 0 & 0 & 0 \\
0 & 0 & 1 / 2 & 0 & 0 & 0 & 0 & 0 & 0 & 0 & 0 & 0 & 0 & 0 & 0 & 0 \\
1 & 0 & 0 & 1 & 0 & 0 & 0 & 0 & 0 & 0 & 0 & 0 & 0 & 0 & 0 & 0 \\
0 & 1 & 0 & 0 & 1 & 0 & 0 & 0 & 0 & 0 & 0 & 0 & 0 & 0 & 0 & 0 \\
0 & 0 & 1 & 0 & 0 & 1 & 0 & 0 & 0 & 0 & 0 & 0 & 0 & 0 & 0 & 0 \\
0 & 0 & 0 & 0 & 0 & 0 & 0 & 0 & 0 & 0 & 0 & 0 & 0 & 0 & 0 & 0 \\
0 & 0 & 0 & 0 & 7 & 0 & 0 & 0 & 1 / 2 & 0 & 0 & 0 & 0 & 0 & 0 & 0 \\
0 & 0 & 0 & 1 & 0 & 0 & 0 & 0 & 0 & 1 & 0 & 0 & 0 & 0 & 0 & 0 \\
0 & 0 & 0 & 5 & 5 & 6 & 1 & 0 & 0 & 0 & 0 & 0 & 0 & 0 & 0 & 0 \\
0 & 0 & 0 & 0 & 0 & 0 & 3 & 7 & 3 & 6 & 1 / 2 & 0 & 0 & 0 & 0 & 0 \\
0 & 0 & 0 & 0 & 0 & 0 & 5 & 4 & 7 & 6 & 0 & 1 & 0 & 0 & 0 & 0 \\
0 & 0 & 0 & 0 & 0 & 0 & 6 & 3 & 4 & 7 & 0 & 0 & 0 & 0 & 0 & 0 \\
0 & 0 & 0 & 0 & 0 & 0 & 0 & 0 & 0 & 0 & 1 & 0 & 0 & 0 & 0 & 0 \\
0 & 0 & 0 & 0 & 0 & 0 & 0 & 0 & 0 & 0 & 0 & 1 & 0 & 0 & 1 & 0 \\
0 & 0 & 0 & 0 & 0 & 0 & 0 & 0 & 0 & 0 & 0 & 0 & 1 & 0 & 0 & 1
\end{array}\right]
$$

Then $S=A_{0}+A_{1}$ has spectral radius $1, \lambda(S)=(3,3,1,3,3), \eta(S)=(4,4,2,2,1)$ and $\eta\left(\operatorname{Adj}\left(G\left(A_{0}, A_{1}\right)\right)=(4,3,3,2,1)\right.$. Note that $\operatorname{dim} \operatorname{ker}\left(\left(1-\operatorname{Adj}\left(G\left(A_{0}, A_{1}\right)\right)\right)^{m}\right)=$ $\operatorname{dim} \operatorname{ker}\left(\left(1-C_{L}\right)^{m}\right)$ for $m=0,1,2, \ldots$, where $C_{L}$ is the companion matrix of the matrix polynomial $L(z)=z^{2}-z A_{1}-A_{0}$; see [6, Prop. 7]. This remark may help to check the example.

QUESTION 5.6. Let $\eta$ be a finite nonincreasing sequence of positive integers. Does there exist a nonnegative square matrix $S$ and an integer $l \geq 1$ such that for all finite sequences $\lambda$ with the same number of elements, positive integers as elements 
and which is majorized by $\eta$ in the sense mentioned above there exist nonnegative matrices $A_{0}, \ldots, A_{l}$ with

$$
S=A_{0}+\ldots+A_{l} \quad \text { and } \quad \lambda\left(\operatorname{Adj}\left(G\left(A_{0}, \ldots, A_{l}\right)\right)=\lambda \quad ?\right.
$$

Acknowledgements. We are indebted to the referee for his/her very careful reading of the manuscript and for constructive remarks and suggestions.

\section{REFERENCES}

[1] B. Bollobás. Modern Graph Theory. Springer, New York, 1998.

[2] L. Elsner, M. Neumann, and B. Vemmer. The Effect of the Number of Processors on the Convergence of the Parallel Jacobi Method. Linear Algebra and its Applications, 154/156:311-330, 1991.

[3] K.-H. Förster and B. Nagy. Some Properties of the Spectral Radius of a Monic Operator Polynomial with Nonnegative Compact Coefficients. Integral Equations and Operator Theory, 14:794-805, 1991.

[4] K.-H. Förster and B. Nagy. Spektraleigenschaften von Matrix- und Operatorpolynomen. Sitzungsberichte der Berliner Mathematischen Gesellschaft, 243-262, 1994.

[5] K.-H. Förster and B. Nagy. Local Spectral Radii and Collatz-Wielandt Numbers of Monic Operator Polynomials with Nonnegative Coefficients. Linear Algebra and its Applications, 268:41-57, 1998

[6] K.-H. Förster and B. Nagy. On Spectra of Expansion Graphs and Matrix Polynomials, to appear in Linear Algebra and its Applications.

[7] S. Friedland and H. Schneider. Spectra of Expansion Graphs. Electronic Journal of Linear Algebra, 6:2-10, 1999.

[8] D. Hershkowitz. The Combinatorial Structure of Generalized Eigenspaces - from Nonnegative Matrices to General Matrices. Linear Algebra and its Applications, 302-303:173-191, 1999.

[9] H. Minc. Nonnegative Matrices. John Wiley \& Sons, New York, 1988.

[10] R.T. Rau. On the Peripheral Spectrum of a Monic Operator Polynomial with Positive Coefficients. Integral Equations and Operator Theory, 15:479-495, 1992.

[11] U.G. Rothblum. Algebraic Eigenspaces of Nonnegative Matrices. Linear Algebra and its Applications, 12:281-292, 1975.

[12] H. Schneider. The Influence of the Marked Reduced Graph of a Nonnegative Matrix on the Jordan form and Related Properties: A Survey. Linear Algebra and its Applications, 84:161$189,1986$. 